\title{
Comparative Evaluation of Echinococcus Serology with Cytology for the Diagnosis of Hepatic Hydatid Disease
}

\author{
Supriya Mahajan ${ }^{1}$ Shalini Thapar ${ }^{2}$ Vikas Khillan ${ }^{3}$ Pradheer Gupta ${ }^{1} \quad$ Archana Rastogi $^{4} \quad$ Ekta Gupta ${ }^{1}$
}

${ }^{1}$ Department of Clinical Virology, Institute of Liver and Biliary Sciences, Delhi, India

2Department of Radiology, Institute of Liver and Biliary Sciences, Delhi, India

${ }^{3}$ Department of Microbiology, Institute of Liver and Biliary Sciences, Delhi, India

${ }^{4}$ Department of Pathology, Institute of Liver and Biliary Sciences, Delhi, India

J Lab Physicians:2020;12:98-102

\author{
Address for correspondence Ekta Gupta, MBBS, MD, \\ Department of Clinical Virology, Institute of Liver and \\ Biliary Sciences, D-1, Vasant Kunj, New Delhi 110070, India \\ (e-mail: ektagaurisha@gmail.com).
}

\section{Abstract}

Keywords

- hepatic hydatid disease

- ELISA

- fine needle aspiration cytology

- liver mass lesions
Objectives The purpose of this study is to determine the diagnostic efficacy of enzyme-linked immunosorbent assay (ELISA) in radiologically confirmed liver mass lesions for the diagnosis of hepatic hydatid disease (HHD) and to compare the diagnostic performance of ELISA with fine needle aspiration cytology (FNAC) (taken as standard) for HHD diagnosis.

Materials and Methods This retrospective study included blood samples of 223 patients with radiologically confirmed liver mass lesions in which immunoglobulin $G(\mathrm{IgG})$ anti-Echinococcus antibodies were tested using a commercial IgG ELISA (RIDASCREEN, R-Biopharm AG, Darmstadt, Germany). Results of ELISA, ultrasonography, FNAC, and liver function tests were obtained from the hospital information system. ELISA results were compared with those of FNAC to analyze the diagnostic efficacy of ELISA for HHD diagnosis.

Statistical Analysis Comparison of the results obtained from ELISA was performed with respect to FNAC results (taken as standard) to analyze the diagnostic efficacy of ELISA for HHD detection. Data has been represented as median (range) or in frequencies. Wilson score was used to assess $95 \%$ confidence interval of diagnostic parameters. The analysis was performed using SPSS Version 22.0 (IBM Corp.) and Open Epi (version 3.01).

Results Out of 223 cases with liver mass lesions, Echinococcus IgG was reactive in $62(28 \%)$ cases and FNAC was positive in $16(7.2 \%)$ cases. Since two cases were FNACpositive but IgG-nonreactive, total HHD cases were 64 (28.7\%). Echinococcus IgG reactive cases were seen more in the extremes of age group, that is, 1 to 10 years and 81 to 90 years. Taking FNAC as the standard, the sensitivity, specificity, positive predictive value, and negative predictive value of ELISA were 87.5, 76.8, 22.6, and 98.7\%, respectively. Cytology-positive cases demonstrated a mean ELISA optical density/ cut-off (OD/CO) of $4.2 \pm 3$ standard deviation.

Conclusion ELISA in radiologically confirmed liver mass cases is highly sensitive in detecting HHD and hence should be used along with ultrasonography for the screening of HHD followed by confirmation with cytology even in cases with a higher OD/CO of ELISA. (c) 2020 by The Indian Association of Laboratory Physicians
License terms

(이 (1) $\Theta \circledast$ 


\section{Introduction}

Echinococcosis or hydatid disease (HD) is a parasitic illness caused by infection with dog tapeworms (Echinococcus granulosus and Echinococcus multilocularis) in its larval stage. ${ }^{1} \mathrm{HD}$ global prevalence is estimated at 2 to 3 million human cases, with an estimated mortality rate of 2 to $4 \%$ per 100 inhabitants. ${ }^{2,3} \mathrm{HD}$ is endemic in more than 100 countries globally, with the highest prevalence found in Mediterranean regions, parts of Russia, central Asia, China, Australia, parts of South America, and Africa. It has been included in the list of neglected tropical diseases and is considered to be one of the six priority neglected zoonotic diseases. ${ }^{4}$ Although HD is endemic in many regions in India, detailed epidemiological studies from India are scanty. ${ }^{4,5}$ The parasite exhibits a predilection for the liver (70-80\%) followed by the lungs (20-30\%), with the spleen, kidney, heart, brain, bone, and breasts being involved less commonly. ${ }^{6,7}$ The definitive host for the parasite is dogs, whereas animals such as sheep, cattle, goats, and camels serve as intermediate hosts. ${ }^{8}$ Humans are deadend occasional intermediate hosts and acquire the infection through accidental ingestion of Echinococcus eggs excreted with feces in dogs and transmitted through a close contact with infected animals. ${ }^{9,10}$

Most HD patients either remain asymptomatic for several years or exhibit nonspecific symptoms due to which diagnosis is often made incidentally. ${ }^{11}$ Infection with Echinococcus induces an antibody response, most commonly immunoglobulin (Ig) G followed by IgM, IgA, and IgE. ${ }^{12}$ Hence, the main serological methods used for human HD diagnosis and follow-up are based on the detection of specific IgG antibodies. The usual diagnostic approach to HD involves a combination of both imaging (ultrasonography [USG], computed tomography [CT], magnetic resonance imaging [MRI]) and serological techniques (IgG enzyme-linked immunosorbent assay [ELISA], indirect fluorescent antibody [IFA], indirect hemagglutination [IHA], or latex agglutination [LA] $)^{4}$ in conjunction with a history of exposure or immigration from an endemic area. ${ }^{12,13}$ The definitive diagnosis of HD can be achieved by the demonstration of scolices, hooklets, or protoscolices in aspirated fluid by fine needle aspiration cytology (FNAC), but it is typically not performed in cases with a suspicion of hydatid cyst due to the perceived risk of anaphylactic shock. ${ }^{4,11,14}$ In routine clinical practices, most clinicians usually rely on the modern imaging techniques for HD diagnosis ${ }^{8,9}$ because although serology is a helpful diagnostic adjunct, its efficacy as a screening assay for HD diagnosis is still debatable. ${ }^{15,16}$

Hence, the aim of this study was to determine the diagnostic efficacy of ELISA in radiologically confirmed liver mass lesions for the diagnosis of hepatic HD (HHD) and to compare the diagnostic performance of ELISA with FNAC (taken as standard) for HHD diagnosis.

\section{Materials and Methods}

\section{Study Population}

This is a retrospective study that included 223 patients with radiologically confirmed liver mass lesions whose blood samples were received, serum separation was performed, and Echinococcus IgG serology was performed in the Department of Clinical Virology in a tertiary liver-care hospital in Delhi from August 2014 to July 2017. The Institutional Ethics Committee (IEC) of the Institute of Liver and Biliary Sciences approved the study protocol.

\section{Serology}

Patients were tested for IgG anti-Echinococcus antibodies by commercial IgG ELISA (RIDASCREEN, R-Biopharm AG, Darmstadt, Germany) as per the manufacturer's instructions. This test is an enzyme immunoassay for the qualitative determination of IgG antibodies against Echinococcus granulosus and Echinococcus multilocularis in human serum. Results of Echinococcus IgG serology was obtained from the hospital information system (HIS).

\section{Data Gathering}

The following other parameters were retrieved from HIS: USG findings, FNAC reports, and liver function tests (LFTs).

\section{Statistical Analysis}

Comparison of the results obtained from ELISA was performed with respect to FNAC results (taken as standard) to analyze the diagnostic efficacy of ELISA for HHD detection. Data have been represented as median (range) or frequencies. The Wilson score was used to assess 95\% confidence interval of diagnostic parameters. The analysis was performed using SPSS Version 22.0 (IBM Corp.) and Open Epi (version 3.01).

\section{Results}

A total of 223 cases having a median age of 50 years (range: 4-85 years) with radiologically confirmed liver mass lesions were included in the study. Median LFTs were found to be normal in the study population, as shown in - Table 1, with cystic lesions of the liver being the most predominant (48.4\%) among the liver mass lesions.

Out of 223 cases with liver mass lesions, Echinococcus IgG was found to be reactive in $62(28 \%)$ cases and nonreactive in 161 (72\%) cases. LFTs were deranged in only $17 / 62(27.4 \%)$ serologically confirmed HHD cases, with a median ALT (alanine aminotransferase) of $26 \mathrm{IU} / \mathrm{mL}$ (range: 9-660 IU/mL) and a median AST (aspartate aminotransferase) of $29 \mathrm{IU} / \mathrm{mL}$ (range: 11-295 IU/mL). Among the radiologically confirmed liver mass lesions in the 62 Echinococcus IgG reactive cases; the most predominant was hepatic cyst $(63 \%)$ followed by liver abscess (22.5\%) and solid mass lesion (14.5\%).

The study group showed the maximum number of liver mass lesions in the age group of 51 to 60 years. Echinococcus $\operatorname{IgG}$ reactive cases were seen more in the extremes of age-group, that is, 1 to 10 years and 81 to 90 years. Both the radiologically confirmed liver mass lesions and serologically confirmed liver hydatid cases demonstrated male preponderance except IgG reactive cases in the age group of 41 to 50 years, which demonstrated female preponderance (-Table 2 ).

Of the 223 cases with liver mass lesions, FNAC was positive in $16(7.2 \%)$ cases, and of the 62 serologically confirmed 
HHD cases, FNAC was positive in only 14 (22.6\%) cases. There were two cases that were FNAC-positive but IgG-nonreactive. Hence, the total HHD cases in this study were 64/223 (28.7\%).

Out of 16 FNAC-positive cases, 14 (87.5\%) were found to be IgG-reactive. Overall, both FNAC and ELISA were positive in $14 / 223$ (6.3\%) cases and negative in 159/223 (71.3\%) cases. Hence, the concordance rate between ELISA and FNAC results for the diagnosis of HHD cases was 77.6\% (- Tables 3 and 4).

- Table 5 depicts the diagnostic performance of ELISA as compared with FNAC for HHD diagnosis.

Among the 62 ELISA reactive cases, a maximum number of samples demonstrated a low optical density/cutoff (OD/ $\mathrm{CO}$ ) in the range of 1 to 2 (-Table 6 ), with a mean OD/CO of $3.28 \pm 2$ standard deviation (SD). Maximum cytology-positive

Table 1 Baseline characteristics of the study population

\begin{tabular}{|l|l|}
\hline $\begin{array}{l}\text { Total number of samples with radiologically } \\
\text { confirmed liver mass lesions }(n)\end{array}$ & 223 \\
\hline Age (years), median (range) & $50(4-85)$ \\
\hline Male, $n(\%)$ & $146(65.5 \%)$ \\
\hline Female, $n(\%)$ & $77(34.5 \%)$ \\
\hline Male:female & $1.9: 1$ \\
\hline ALT (IU/L), median (range) & $25.5(7-717)$ \\
\hline AST (IU/L), median (range) & $29(8-671)$ \\
\hline Bilirubin (total) (mg/dL), median (range) & $0.6(0.2-25.3)$ \\
\hline $\begin{array}{l}\text { Total number of radiologically confirmed liver } \\
\text { cystic lesions }\end{array}$ & $108 / 223(48.4 \%)$ \\
\hline $\begin{array}{l}\text { Total number of radiologically confirmed liver } \\
\text { abscess lesions }\end{array}$ & $79 / 223(35.4 \%)$ \\
\hline $\begin{array}{l}\text { Total number of radiologically confirmed liver } \\
\text { solid mass lesions }\end{array}$ & $36 / 223(16.14 \%)$ \\
\hline
\end{tabular}

Abbreviations: ALT, alanine aminotransferase; AST, aspartate aminotransferase. cases were seen in cases with $\mathrm{OD} / \mathrm{CO}>10$ followed by those in the range of 4 to 5 . The 16 cytology-positive cases demonstrated a mean ELISA OD/CO of $4.2 \pm 3$ SD.

\section{Discussion}

Echinococcosis is among the most neglected parasitic diseases, and most clinicians usually rely on the modern imaging techniques for its diagnosis, with USG being the method of choice for the detection of both hepatic and extrahepatic echinococcosis..$^{8,9}$ Although HD serology is a helpful diagnostic adjunct, its efficacy as a screening assay for HD diagnosis is still debatable. ${ }^{15,16}$ This may be because in developing countries like India, serological tests for HD, which are still considered as alternatives to imaging modalities, are not routinely employed in clinical settings. ${ }^{12}$ Hence, this study aimed at determining the diagnostic efficacy of ELISA as a screening assay in radiologically confirmed liver mass lesions for the diagnosis of HHD and to compare the diagnostic performance of ELISA with FNAC (taken as standard) for HHD diagnosis.

In the study, ELISA was reactive in 62 (28\%) USGpositive HD cases, which is in accordance with a study from Chandigarh in North India ${ }^{17}$ but in contrast to studies from China and Egypt where seropositivity was higher. ${ }^{18,19}$ On the other hand, FNAC in the study was positive in 16 (7.2\%) cases, which is similar to two other studies from Chandigarh. ${ }^{20,21}$

In the study, Echinococcus IgG reactive cases were seen more in the age group of 1 to 20 years with a male preponderance, whereas liver mass lesions on USG were predominant in the age group of 51-60 years. This result is in accordance with a seroepidemiological study performed in Kashmir (North India) where age < 15 years and male gender were considered as significant factors associated with Echinococcus seropositivity, ${ }^{5}$ thus supporting the worldwide earlier well-reported observation that infection is usually

Table 2 Age- and sex-wise distribution of radiologically confirmed liver mass lesions $(n=223)$ and serologically confirmed liver hydatid cases $(n=62)$

\begin{tabular}{|c|c|c|c|c|c|}
\hline Age (years), $n$ & $\begin{array}{l}\text { Total no. of males } \\
\text { with liver mass } \\
\text { lesions, } n(\%)\end{array}$ & $\begin{array}{l}\text { Total no. of } \\
\text { females with liver } \\
\text { mass lesions, } n \\
(\%)\end{array}$ & $\begin{array}{l}\text { Total no. of IgG- } \\
\text { reactive cases, } \\
n(\%)\end{array}$ & $\begin{array}{l}\text { Total no. of IgG- } \\
\text { reactive (males), } \\
n(\%)\end{array}$ & $\begin{array}{l}\text { Total No. of } \\
\text { lgG-reactive } \\
\text { (females), } n(\%)\end{array}$ \\
\hline $1-10(5)$ & $3(60)$ & $2(40)$ & $3(60)$ & $2(66.7)$ & $1(33.3)$ \\
\hline $11-20(11)$ & $6(54.5)$ & $5(45.4)$ & $6(54.5)$ & $3(50)$ & $3(50)$ \\
\hline $21-30(20)$ & $10(50)$ & $10(50)$ & $6(30)$ & $3(50)$ & $3(50)$ \\
\hline $31-40$ (35) & $26(74.3)$ & 9 (25.7) & $14(40)$ & $8(57)$ & $6(43)$ \\
\hline $41-50(44)$ & $28(63.6)$ & $16(36.4)$ & $15(34 \%)$ & $7(46.7)$ & $8(53.3)$ \\
\hline $51-60(55)$ & $34(61.8)$ & $21(38.2)$ & $8(14.5)$ & $4(50)$ & $4(50)$ \\
\hline $61-70(22)$ & $17(77.3)$ & $5(22.7)$ & $1(4.5)$ & $0(0)$ & $1(100)$ \\
\hline $71-80(28)$ & $20(71.4)$ & $8(28.6)$ & $7(25)$ & $5(71.4)$ & $2(28.6)$ \\
\hline $81-90(3)$ & $2(66.7)$ & $1(33.3)$ & $2(66.7)$ & $2(100)$ & $0(0)$ \\
\hline Total $=223$ & $146(65.5)$ & $77(34.5)$ & $62(28)$ & $34(55)$ & $28(45)$ \\
\hline
\end{tabular}


Table 3 Comparative analysis of FNAC and ELISA results for the diagnosis of hepatic hydatid cases

\begin{tabular}{|l|l|l|l|l|}
\hline \multicolumn{2}{|c|}{} & \multicolumn{2}{|c|}{ Total } \\
\cline { 3 - 6 } \multicolumn{2}{|c|}{ ELISA } & Reactive & Positive & Negative \\
\hline & Nonreactive & 14 & 48 & 62 \\
\hline & Total & 2 & 159 & 161 \\
\hline
\end{tabular}

Abbreviations: ELISA, enzyme-linked immunosorbent assay; FNAC, fine needle aspiration cytology.

Table 4 Results obtained from ELISA and cytology in radiologically confirmed liver mass lesions $(n=223)$

\begin{tabular}{|l|l|}
\hline & Total, $\boldsymbol{n}$ (\%) \\
\hline Total ELISA-reactive & $62(28)$ \\
\hline Total FNAC-positive & $16(7.2)$ \\
\hline ELISA-reactive and FNAC-positive & $14(6.3)$ \\
\hline ELISA-reactive and FNAC-negative & $48(21.5)$ \\
\hline ELISA-nonreactive and FNAC-positive & $2(0.9)$ \\
\hline $\begin{array}{l}\text { Total cases of hepatic hydatid disease by either } \\
\text { ELISA or FNAC or both }\end{array}$ & $64(28.7)$ \\
\hline
\end{tabular}

Abbreviations: ELISA, enzyme-linked immunosorbent assay; FNAC, fine needle aspiration cytology.
Table 5 Diagnostic performance of ELISA as compared with FNAC for the diagnosis of liver hydatid cases

\begin{tabular}{|l|l|}
\hline Sensitivity $(95 \% \mathrm{Cl})$ & $87.5 \%(63.98-96.50)$ \\
\hline Specificity $(95 \% \mathrm{Cl})$ & $76.8 \%(70.61-82.04)$ \\
\hline Positive predictive value $(95 \% \mathrm{Cl})$ & $22.6 \%(13.96-34.41)$ \\
\hline Negative predictive value $(95 \% \mathrm{Cl})$ & $98.7 \%(95.58-99.66)$ \\
\hline Diagnostic accuracy $(95 \% \mathrm{Cl})$ & $77.58 \%(71.66-82.56)$ \\
\hline False-positive rate & $21.5 \%$ \\
\hline False-negative rate & $0.89 \%$ \\
\hline
\end{tabular}

Abbreviations: $\mathrm{Cl}$, confidence interval; ELISA, enzyme-linked immunosorbent assay; FNAC, fine needle aspiration cytology.

Table 6 Correlation of FNAC results with ELISA OD/CO results in radiologically confirmed liver mass lesions ( $n=223$ )

\begin{tabular}{|l|l|l|l|}
\hline ELISA result interpretation & ELISA OD/CO $(\boldsymbol{n})$ & FNAC-positive, $\boldsymbol{n}(\%)$ & FNAC-negative, $\boldsymbol{n}(\%)$ \\
\hline \multirow{4}{*}{ Nonreactive } & $<0.9(160)$ & $2(1.3)$ & $158(98.7)$ \\
\hline & $1-2(22)$ & $1(4.5)$ & $21(95.5)$ \\
\cline { 2 - 4 } & $2-3(14)$ & $2(14.3)$ & $12(85.7 \%)$ \\
\cline { 2 - 4 } & $3-4(9)$ & $5(62.5)$ & $7(77.8)$ \\
\cline { 2 - 4 } & $4-5(8)$ & $1(33.3)$ & $3(37.5)$ \\
\cline { 2 - 4 } & $5-6(3)$ & $0(0)$ & $2(66.7)$ \\
\cline { 2 - 4 } & $6-7(1)$ & $1(33.3)$ & $2(100)$ \\
\cline { 2 - 4 } & $7-8(3)$ & $1(50)$ & $1(50)$ \\
\cline { 2 - 4 } & $8-9(2)$ & $0(0)$ & $0(0)$ \\
\cline { 2 - 4 } & $9-10(0)$ & $1(100)$ & $0(0)$ \\
\cline { 2 - 4 } & $>10(1)$ & $16(7)$ & $207(93)$ \\
\hline
\end{tabular}

Abbreviations: ELISA, enzyme-linked immunosorbent assay; FNAC, fine needle aspiration cytology; OD/CO, optical density/cutoff.

acquired in childhood, remaining asymptomatic for long period, with symptoms manifesting in adult age depending upon the organ involved. ${ }^{22}$

In this study, ELISA demonstrated a fairly good sensitivity and negative predictive value of 87.5 and $98.7 \%$, respectively, but a moderately low specificity of $76.8 \%$ when FNAC was taken as the standard in all radiologically confirmed liver mass lesions. Other studies have usually evaluated the diagnostic efficacy of ELISA using imaging techniques especially USG, with a sensitivity of ELISA ranging from 64.8 to $100 \%{ }^{2,18}$ Another study found that the sensitivity of ELISA was $100 \%$ when compared with IHA, IFA, and Casoni's intradermal test. ${ }^{23}$ To the best of our knowledge, this is the first study that has evaluated the diagnostic efficacy of ELISA with FNAC for HHD diagnosis taking into consideration the fact that FNAC is a confirmatory test for HHD diagnosis ${ }^{4,24}$ in contrast to USG, which although is a convenient tool for HHD diagnosis and detects the location, number, and size of the cysts with relative ease $\mathrm{e}^{22,25-27}$ but has poor sensitivity in detecting small-sized cysts, ${ }^{11,15}$ and USG findings may mimic other pathologies presenting as liver mass lesions, ${ }^{4}$ which has also been found in this study as only $62 / 223$ (28\%) of the liver mass lesions on USG demonstrated IgG reactivity.

Interestingly, when $\mathrm{OD} / \mathrm{CO}$ results of ELISA were compared with FNAC results, it was found that as the OD/CO increases from 1 to 5 , FNAC positivity demonstrated $58 \%$ increase from $4.5 \%$ to $62.5 \%$, but as the $\mathrm{OD} / \mathrm{CO}$ increased beyond 5 , FNAC 
positivity showed a sudden decline. The 16 cytology-positive cases demonstrated mean ELISA OD/CO of $4.2 \pm 3$ SD with maximum cytology-positive cases seen with $\mathrm{OD} / \mathrm{CO}>10$ followed by those in the range of 4 to 5 . This is the first study that has compared the OD/CO of ELISA with FNAC results for the diagnosis of HHD. Since no OD/CO ranges taken in the study showed $100 \%$ FNAC positivity, it can be speculated that all ELISA results of USG-positive cases must be confirmed by FNAC irrespective of OD/CO range.

\section{Limitations of the Study}

Follow-up of the patients could not be performed as this was a retrospective study.

\section{Conclusion}

In conclusion, the diagnostic approach of HHD should involve a combination of imaging techniques, serological tests, and cytology, as no single diagnostic modality is enough to arrive at a confirmation of the presence of HHD. ELISA in radiologically confirmed liver mass cases is highly sensitive in detecting HHD and hence should be used along with USG for the screening of HHD followed by confirmation with cytology even in cases with a higher OD/CO of ELISA.

\section{Funding}

This research did not receive any specific grant from funding agencies in the public, commercial, or profit sectors.

\section{Conflicts of Interest}

There are no conflicts of interest to report.

\section{References}

1 Li H, Song T, Shao Y, Aili T, Ahan A, Wen H. Comparative evaluation of liposomal Albendazole and tablet-Albendazole against hepatic cystic echinococcosis: a non-randomized clinical trial. Medicine (Baltimore) 2016;95(4):e2237

2 Manzano-Román R, Sánchez-Ovejero C, Hernández-González A, Casulli A, Siles-Lucas M. Serological diagnosis and follow-up of human cystic echinococcosis: a new hope for the future? BioMed Res Int 2015;2015:428205

3 Belhassen-García M, Romero-Alegria A, Velasco-Tirado V, et al. Study of hydatidosis-attributed mortality in endemic area. PLoS One 2014;9(3):e91342

4 Malla N, Mewara A. Human cystic echinococcosis with special reference to India - an overview. Ann Clin Cytol Pathol 2016;2(5):1038

5 Fomda BA, Khan A, Thokar MA, et al. Sero-epidemiological survey of human cystic echinococcosis in Kashmir, North India. PLoS One 2015;10(4):e0124813

6 Wuestenberg J, Gruener B, Oeztuerk S, et al. Diagnostics in cystic echinococcosis: serology versus ultrasonography. Turk J Gastroenterol 2014;25(4):398-404

7 Derfoufi O, Ngoh Akwa E, Elmaataoui A, et al. Epidemiological profile of echinococcosis in Morocco from 1980 to 2008 [in French]. Ann Biol Clin (Paris) 2012;70(4):457-461
8 Mandal S, Mandal MD. Human cystic echinococcosis: epidemiologic, zoonotic, clinical, diagnostic and therapeutic aspects. Asian Pac J Trop Med 2012;5(4):253-260

9 Piccoli L, Tamarozzi F, Cattaneo F, et al. Long-term sonographic and serological follow-up of inactive echinococcal cysts of the liver: hints for a "watch-and-wait" approach. PLoS Negl Trop Dis 2014;8(8):e3057

10 Ernest E, Nonga HE, Kynsieri N, Cleaveland S. A retrospective survey of human hydatidosis based on hospital records during the period 1990-2003 in Ngorongoro, Tanzania. Zoonoses Public Health 2010;57(7-8):e124-e129

11 Mihmanli M, Idiz UO, Kaya C, et al. Current status of diagnosis and treatment of hepatic echinococcosis. World J Hepatol 2016;8(28):1169-1181

12 Pakala T, Molina M, Wu GY. Hepatic echinococcal cysts: a review. J Clin Transl Hepatol 2016;4(1):39-46

13 Symeonidis N, Pavlidis T, Baltatzis M, et al. Complicated liver echinococcosis: 30 years of experience from an endemic area. Scand J Surg 2013;102(3):171-177

14 Siracusano A, Delunardo F, Teggi A, Ortona E.. Host-parasite relationship in cystic echinococcosis: an evolving story. Clin Dev Immunol 2012;2012-639362

15 McManus DP, Gray DJ, Zhang W, Yang Y. Diagnosis, treatment, and management of echinococcosis. BMJ 2012;344:e3866

16 Brunetti E, Garcia HH, Junghanss T; International CE Workshop in Lima, Peru, 2009. Cystic echinococcosis: chronic, complex, and still neglected. PLoS Negl Trop Dis 2011;5(7):e1146

17 Khurana S, Das A, Malla N. Increasing trends in seroprevalence of human hydatidosis in North India: a hospital-based study. Trop Doct 2007;37(2):100-102

18 Yang YR, Craig PS, Ito A, et al. A correlative study of ultrasound with serology in an area in China co-endemic for human alveolar and cystic echinococcosis. Trop Med Int Health 2007;12(5):637-646

19 Abdelraouf A, El-Aal AA, Shoeib EY, et al. Clinical and serological outcomes with different surgical approaches for human hepatic hydatidosis. Rev Soc Bras Med Trop 2015;48(5):587-593

20 Gochhait D, Dey P, Rajwanshi A, Nijhawan R, Radhika S, Gupta N. Spectrum of fungal and parasitic infections on fine needle aspiration cytology. Diagn Cytopathol 2015;43(6):450-455

21 Bakshi P, Srinivasan R, Rao KL, et al. Fine needle aspiration biopsy in pediatric space-occupying lesions of liver: a retrospective study evaluating its role and diagnostic efficacy. J Pediatr Surg 2006;41(11):1903-1908

22 Moro P, Schantz PM. Echinococcosis: a review. Int J Infect Dis 2009;13(2):125-133

23 Wattal C, Malla N, Khan IA, Agarwal SC. Comparative evaluation of enzyme-linked immunosorbent assay for the diagnosis of pulmonary echinococcosis. J Clin Microbiol 1986;24(1):41-46

24 Brunetti E, Junghanss T. Update on cystic hydatid disease. Curr Opin Infect Dis 2009;22(5):497-502

25 Zhang W, McManus DP. Recent advances in the immunology and diagnosis of echinococcosis. FEMS Immunol Med Microbiol 2006;47(1):24-41

26 Pawlowski ZS, Eckert DA, Vuitton DA, et al. Echinococcosis in humans: clinical aspects, diagnosis and treatment. In: Eckert J, Gemmell MA, Meslin F-X, Pawlowski ZS, eds. WHO/OIE Manual on Echinococcosis in Humans and Animals: A Public Health Problem of Global Concern. Paris: World Organisation for Animal Health; 2001 20-72

27 Nunnari G, Pinzone MR, Gruttadauria S, et al. Hepatic echinococcosis: clinical and therapeutic aspects. World J Gastroenterol 2012;18(13):1448-1458 